Europhys. Lett., 42 (3), pp. 247-252 (1998)

\title{
Discrimination of deterministic dynamics in the spontaneous activity of the human brain cortex
}

\author{
L. Narici $(*)$, S. Boccaletti $(* *)$, A. Giaquinta $\left({ }^{* *}\right)$ and F. T. Arecchi $\left({ }^{* *}\right)$ \\ Istituto Nazionale di Ottica, 50125 Firenze, Italy \\ (received 16 December 1997; accepted 17 March 1998) \\ PACS. 05.45+b - Theory and models of chaotic systems. \\ PACS. 87.40+w - Biomagnetism (cardiomagnetism, neuromagnetism, tissue susceptibility) and \\ magnetobiology (magnetic field effects on cellular processes, and on naviga- \\ tion and behavior of various species). \\ PACS. 87.22-q - Physics of cellular and physiological processes.
}

\begin{abstract}
We apply a new strategy to isolate cortical rhythms as deterministic signals and discuss several related topics. Extraction of the relevant signal from the original data is achieved by a novel use of the localized filtering action of the wavelet transform. Alternative nonlinear data analysis tools are used to test the validity of the procedure. In the case of neuromagnetic signals measured on the occipital cortex of a normal subject, we demonstrate the existence of a deterministic activity within the $\alpha$-band $(8-12 \mathrm{~Hz})$.
\end{abstract}

The study of the brain bioelectrical activities in terms of deterministic nonlinear dynamics is quite recent [1]-[3]. Perspectives of clinical use of the nonlinear tools range from an augmented predictive classification of Alzheimer's desease [4] to the possibility of controlling epileptic activity [5]. However, the nonstationarity of the brain states plus the poor Signal-to-Noise Ratio (SNR) due to the large amount of brain processes active at the same time have forbidden so far a generally accepted procedure to estimate suitable indicators [2], [6], [7]. Whence the need for a suitably tailored filter able to extract the activity under study to avoid contamination between noise and bioelectrical activities while maintaining a sufficient number of data to perform indicator calculations.

The reason for this communication is twofold. We apply for the first time a novel filtering technique based on the local features of the wavelet transformation [8] and, second, we propose a procedure for brain signal processing, making use of available nonlinear tests.

The procedure here suggested estimates the presence of determinism in a magnetoencephalogram using the newly developed $\beta$ test [9], then, whenever allowed, it calculates the correlation dimensions (CD) [10] and tests the validity of the calculation. We will show that, while the measured signal contains deterministic activity, it is not possible to reasonably estimate the $\mathrm{CD}$, for which we find, however, an upper bound of 7 .

$\left({ }^{*}\right)$ Also at Dipartimento di fisica Università di Roma "Tor Vergata", Roma, Italy.

$\left({ }^{* *}\right)$ Also at Department of Physics and Applied Mathematic, Universidad de Navarra, Pamplona, Spain.

$\left({ }^{* * *}\right)$ Also at Dipartimento di Sistemi e Informatica, Università di Firenze, Firenze, Italy.

$\left({ }^{*} *^{*}\right)$ Also at Dipartimento di Fisica, Università di Firenze, 50125 Firenze, Italy.

(C) EDP Sciences 
Our protocol is applied to a normal, relaxed, subject. Spontaneous activity of the brain is measured in the occipital cortex. In these conditions, the signal is more powerful within the $\alpha$-band $(8-12 \mathrm{~Hz}) . \quad \alpha$-rhythm is conceivably less complex than any other cognitive related activity, nevertheless it appears in the ElectroEncephaloGraphy (EEG) and MagnetoEncephaloGraphy (MEG) measurements at irregular intervals. Its understanding might lead to the comprehension of the other bioelectrical cortical phenomena [11]. While the brain signals show activities as fast as $10^{2}-10^{3} \mathrm{~Hz}$, the SNR is often larger than unity only in narrow windows, around $4-5 \mathrm{~Hz}, 10 \mathrm{~Hz}$ ( $\alpha$-band), and sometimes $20 \mathrm{~Hz}$. Furthermore, in those windows, it is not consistently larger than one. As for $\alpha$-rhythm, it is present in bursts (spindles) that appear at irregular intervals. The incidence of spindles may differ, depending on subject and measurement conditions. During each spindle, we have SNR $>1$, but generally at all other times SNR $\ll 1$.

There is no definite general answer to the question about the effects of a filter on the reconstruction of the dynamics underlying the signal. However, in most cases it has been shown that the filtering process affects $\mathrm{CD}$ as well as the other dynamical parameters; even though filtering generally reduces $\mathrm{CD}$, sometimes $\mathrm{CD}$ may even fictitiously increase [12].

We have already noticed that brain signals are nonstationary. The longest stationarity window is found during sleep (anyway less than $10^{3} \mathrm{~s}$ ). For the awake stages ( $\alpha$-rhythm) the stationarity period is estimated to be of the order of $10^{2} \mathrm{~s}$. From the above discussion, it is possible to calculate the maximum value of $\mathrm{CD}$ that one can assign with some confidence.

Let $T$ be the acquisition time interval, $f$ the sampling frequency and $\Delta B$ the filter bandwidth. Filtering in Fourier space, only $N_{\mathrm{F}} \sim T \Delta B$ complex points will be left. With $N=2 T \Delta B$ real independent points in the time domain, assuming $T=10^{2} \mathrm{~s}, \Delta B=4-6 \mathrm{~Hz}$ (as in the $\alpha$-band), the maximum CD that one can estimate is about $2 \log _{10}(N) \sim 6[13]$ or more accurately $2 \log _{10}(N)-2 \sim 4[14]$.

By use of a 28-channel superconductive system [15], we measured for $65 \mathrm{~s}$ the magnetic field generated by the bioelectrical sources over the left occipital cortex of a healthy subject. The subject was lying prone in a bed wearing a blackened rubber mask, watching fixedly two green LEDs to maintain steady eyeball position. The incidence of $\alpha$-spindles was less than $50 \%$. The subject gave consensus to this experiment. The acquisition frequency was $f=1 \mathrm{KHz}$, with a band pass filter $0.4-250 \mathrm{~Hz}$. The results here presented are relative to one channel of the neuromagnetometer, where the presence of the activity in the $\alpha$-band was most preminent. Studies on other channels, also featuring strong $\alpha$-activity, produced similar results.

We apply the embedding technique [16] to the measured time series $x(t)$, thus generating an $m$-dimensional vector $\mathbf{y}(t) \equiv(x(t), x(t-\tau), \ldots, x(t-(m-1) \tau))$, where $\tau$ is the embedding delay time, $m$ the embedding dimension. For a proper choice of $\tau$, we make use of the mutual average information technique [17], which for our data provides $\tau=25$ sampling time intervals.

In order to extract the deterministic part from the embedded data, we apply a recently introduced filtering technique [8], which combines wavelet transform (WT) [18] with an adaptive algorithm for the selection of the filter. For each component $i$ of the embedded vector we consider the variation $\delta x_{i}\left(t_{n+1}\right)=x_{i}\left(t_{n+1}\right)-x_{i}\left(t_{n}\right)$, where $t_{n+1}-t_{n}=\tau_{n}$ is the $n$-th adjustable observation time. The goal of the algorithm is to keep the separation between two successively observed coordinates as stable as possible, by varying the observation time interval (OTI) $\tau_{n}$. For that purpose we consider the local variation rates

$$
\lambda_{i}\left(t_{n+1}\right)=\frac{1}{\tau_{n}} \log \left|\frac{\delta x_{i}\left(t_{n+1}\right)}{\delta x_{i}\left(t_{n}\right)}\right|
$$

and we select a new observation at the time $t_{n+2}=t_{n+1}+\tau_{n+1}$, where $\tau_{n+1}$ is given by

$$
\tau_{n+1}^{i}=\tau_{n}^{i}\left(1-\tanh \left(\sigma \lambda_{i}\left(t_{n+1}\right)\right)\right),
$$




$$
\tau_{n+1}=\min \left[\tau_{n+1}^{i}\right], \quad i=1, \ldots, m ; \sigma>0
$$

The details are given in ref. [9]. By means of the above iterative algorithm it is possible to perform a geometrically regularized observation of the dynamics at irregular times, whose ordering yields some dynamical indicators [9]. A critical parameter in eqs. (1), (2) is the sensitivity $\sigma>0$. It can be considered as a fixed parameter which determines the OTI dynamics by ruling the slope of the tanh function close to zero. However, a more sensible choice [8] consists in introducing a second level of adaptation in the above scheme, and changing $\sigma$ every $L$ OTIs through the rule

$$
\sigma=\sigma_{0}+\frac{m}{M \sum_{i=1}^{m} \sum_{k=1}^{L}\left|\lambda_{i}\left(t_{k}\right)\right|},
$$

where $m$ is the current embedding dimension, $M$ the maximum embedding dimension, $\sigma_{0}>0$ is a minimum sensitivity in case the second term gets close to zero, and the sum runs over all dimensions $(i=1, \ldots, m)$ and over all previous $L$ OTIs $(k=1, \ldots, L)$. Use of eq. (3) leads to a second hierarchical level of adaptation, by introducing a kind of long-term memory, as opposed to the short-term memory represented by the selection process of $\tau$ 's. When $m=M$, eq. (3) reduces to the case already discussed in ref. [9] for a model system with preassigned dimensions.

For an empirical series of data with no a priori information, by use of eqs. (1)-(3), we evaluate the $\beta$ indicator which discriminates between determinism and stochasticity, that is

$$
\beta=\sum_{n}\left|\prod_{i=1}^{m} \lambda_{i}\left(t_{n}\right)\right| .
$$

In ref. [9] it has been demonstrated that the behavior of $\beta$ as a function of the embedding dimension $m$ depends on the nature of the observed process. Namely, if the process is deterministic, then $\beta$ scales as $e^{-m}$ (aside from a factor $\mathcal{O}(1)$ in the exponent), whereas it is a constant for a purely stochastic process. When the considered process is a mixture of a deterministic part and of a stochastic part, then $\beta(m)$ has an exponential decay with an exponent intermediate between 0 and 1. Based upon these considerations, when dealing with a signal which is the sum of a deterministic and a stochastic process, a filter which minimizes the slope of the $\ln \beta(m)$ will extract the deterministic part of the signal. We proceed as follows. We WT transform our data set (via the DAUB20 basis [19]), and then we design a filter $\mathcal{F}(\theta)$ in the WT space which eliminates all those coefficients with absolute values smaller than a given filtering threshold $\theta$. By inverse WT to real space, we obtain the filtered data, which undergo the $\beta$ test. Increasing the value of $\theta$ results in decreasing the slope $\xi$ of $\ln \beta(m)$. However, in the case in which a part of the signal has deterministic nature, the decrease of $\xi$ stops at a saturation value [8], and this allows to define an optimal filtering threshold $\theta_{\text {sat }}$ such that the corresponding filter $\mathcal{F}\left(\theta_{\text {sat }}\right)$ extract as much determinism as possible from the original data set. Figure 1 shows the behavior of $\xi$ as a function of $\theta$ for the analyzed data. Saturation of $\xi$ starts at a threshold $\theta_{\text {sat }}$. Once the optimal filtering threshold has been selected, we analyze the corresponding filtered data set $x^{\prime}$, obtained by inverse WT of the coefficients filtered by $\mathcal{F}\left(\theta_{\text {sat }}\right)$. A negative $\xi$ distinguishes the extracted signal from a stochatic process with no correlations (white noise), but it is not yet sufficient to discriminate a deterministic process from a stochastic process with long correlations (coloured noise) [9]. In order to distinguish $x^{\prime}$ from any coloured noise with the same correlation properties, we calculate the surrogate of $x^{\prime}$ by randomizing the phases of its Fourier components, while keeping the amplitudes unchanged. 


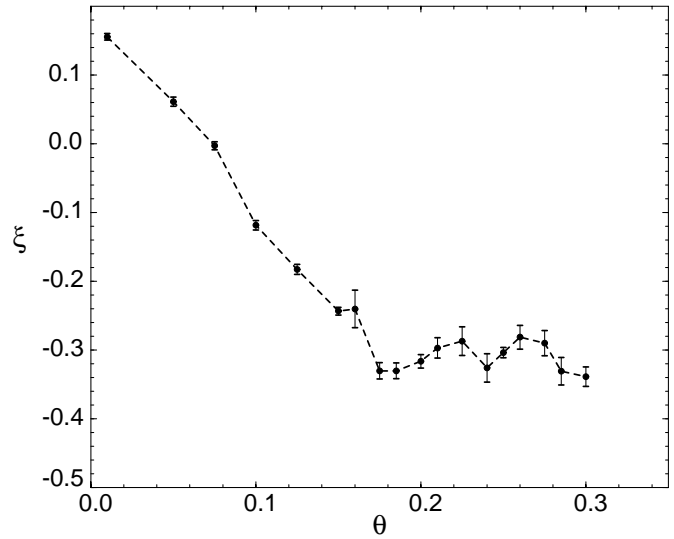

Fig. 1

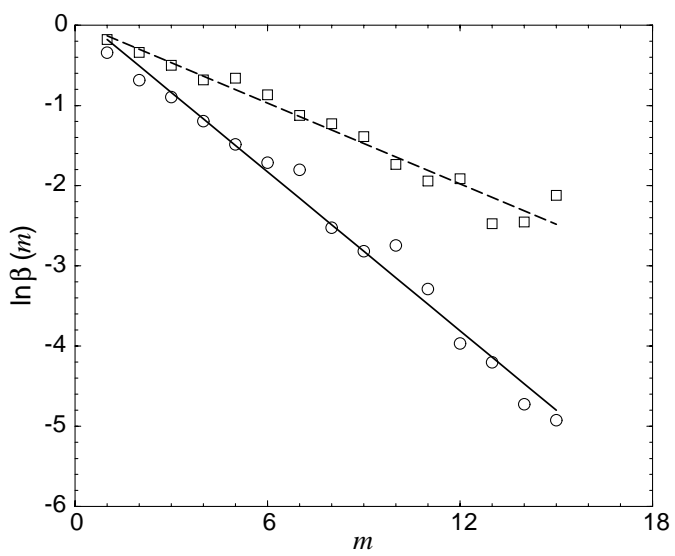

Fig. 2

Fig. 1. - Plot of $\xi$ as a function of the filtering threshold $\theta$ (see text for definitions). $\sigma_{0}=0.0001$, $L=30$. $\xi$ has been calculated as the best linear fit of the $\ln (\beta(m))$ plots for $m=1$ up to $m=15$. The $\xi(\theta)$ behavior indicates the existence of an optimal filtering threshold $\theta_{\text {sat }} \simeq 0.18$.

Fig. 2. $-\ln (\beta)$ as a function of the embedding dimension for $x^{\prime}$ (circles) and for the surrogate of $x^{\prime}$ (squares). Same parameters as in fig. 1. The solid and dashed lines are the best linear fits for the two cases. The slope of the surrogate is intermediate between zero and that of $x^{\prime}$.

This way, we have constructed a noisy signal whose correlation properties are the same as those of $x^{\prime}$ because both signals have the same power spectrum [20].

Figure 2 reports the $\ln \beta(m)$ plot for $x^{\prime}$ and its surrogate, showing that the slope of the surrogate is intermediate between zero and that of $x^{\prime}$. This means that the extracted signal $x^{\prime}$ can be distinguished from any coloured noise featuring the same distribution and autocorrelation function [8]. Thus, our procedure allows to extract a deterministic signal from the MEG data.

The selected filter keeps about 1500 independent data points. Therefore, the upper bound for the calculable CD is 6.4 [13]. Figure 3 reports the filter transfer function. It should be noticed that our filter selects some frequency bands where the determinism is mostly located, while the other frequencies are strongly damped. This feature is peculiar of the combination of the $\beta$ test with the localized action of WT, and indicates the location in frequency of the deterministic part of the MEG signal. At low resolution $(1 \mathrm{~Hz})$ we notice only the generic low-pass character of the filter, whereas at high resolution $(0.5 \mathrm{~Hz})$ the dramatic discrimination of close-by frequencies is evident. This result is very relevant. It can guide further analysis of human brain activities in stimulated cases, where the stimuli are performed within the frequency bands of fig. 3 in order to inspect the response of the system, and possibly to model the corresponding behavior.

We calculate the CD of $x^{\prime}$ by use of the Grassberger and Procaccia algorithm [10] reconstructing the data sets in dimensions $2 \leq m \leq 12$. No reliable saturation is found, as can be seen in fig. 4. This means that the process is high dimensional, and that the number of independent data saved after the filtering action is insufficient for the CD calculation, as discussed above. Anyway, the dimensionality can be estimated by using the algorithm of false nearest neighbors [21]. The results are shown in fig. 5, where the percentage of false neighbors $N(m)$ vanishes at $m=7$ for $x^{\prime}$. Looking at fig. 5 , another important feature appears. The number of false neighbors vanishes at $m=7$ also for the surrogate of $x^{\prime}$, thus indicating that a discrimination between $x^{\prime}$ and a coloured-noise process is impossible via this method. In conclusion, only the $\beta$ method can distinguish $x^{\prime}$ from its surrogate (hence from 


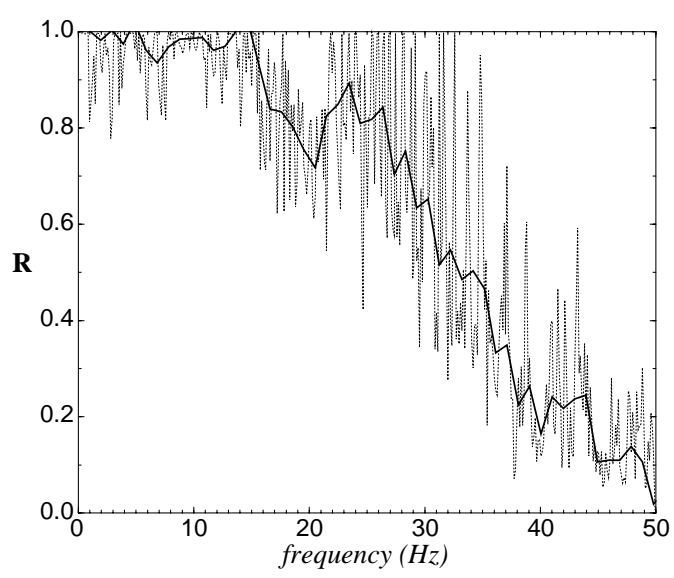

Fig. 3

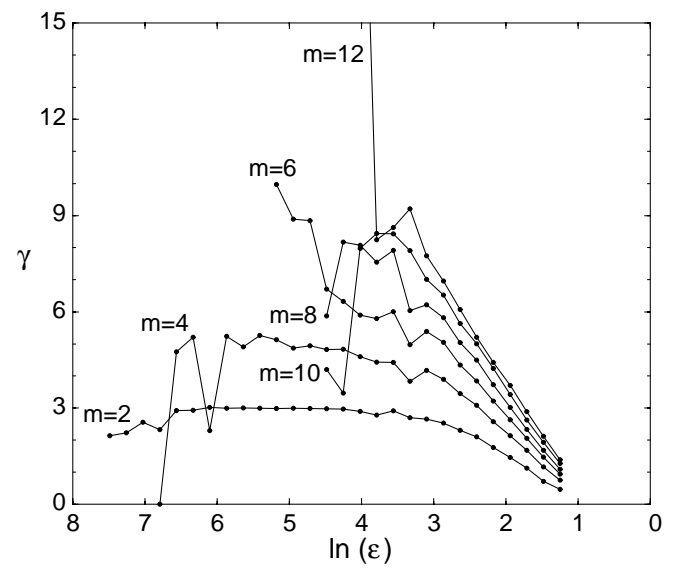

Fig. 4

Fig. 3. - Filter transfer function. For each frequency spanned by the signal, we report the ratio $R$ between the power spectrum of $x^{\prime}$ and that of the original signal (dashed line: $0.5 \mathrm{~Hz}$ resolution; solid line: $1 \mathrm{~Hz}$ resolution).

Fig. 4. - Measurement of CD for $x^{\prime} . \ln (\epsilon)$ is the logarithm of the distance between points, $\gamma$ is the derivative of the function $N(\epsilon)$ (number of points with mutual distance $\epsilon$ ) with respect to $\ln (\epsilon)$. Curves are reported at different embedding dimensions $(m=2,4,6,8,10,12)$. No appreciable saturation is found at increasing $m$.

a coloured-noise process).

Most recognition methods are based upon the distribution of mutual distances in an $m$ dimensional space, thus requiring a number of data scaling exponentially with the embedding dimensions; on the contrary, our strategy exploits the information of a reduced one-dimensional string of data (the OTI sequence), thus requiring a number of data which scales linearly with the embedding dimension [8], [9]. As a consequence, our filter is the natural choice whenever one deals with a high-dimensional process, for which the number of available data is insufficient for those techniques based on the geometry of the time series. Even though we are not able to give a precise assignment of the $\mathrm{CD}$, fig. 5 shows that an upper bound is 7 .

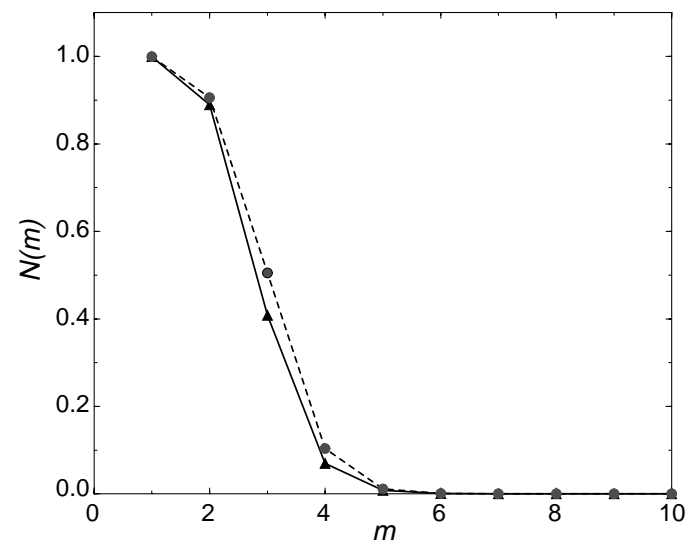

Fig. 5. - Measurement of false neighbors. The percentage $N(m)$ of false nearest neighbors is reported as a function of the embedding dimension $m$. Both for $x^{\prime}$ (triangles and solid line) and for its surrogate (circles and dashed line), the number of false neighbors vanishes at $m=7$. 
In conclusion, we have demonstrated the effectiveness of the adaptive WT filtering technique even when applied to real signals with poor SNR. Our procedure allows to claim the deterministic nature of some of the waking activities of the human cortex. However, the correlation dimension is too high to be calculated. These results may open a new perspective in studying the brain activity in other situations, as, e.g., in stimulated cases, where the signal-to-noise ratio is more favorable and hence the filter influence is weaker. The used technique appears a promising filtering procedure, with a large applicability whenever the process of signal extraction can be assumed to be equivalent to the discrimination of the deterministic component. It presents several advantages with respect to other filters. It is a local filter, i.e. it allows to have different filtering levels at different time instants, a crucial property for those signals, like $\alpha$-activity, which feature SNR greater than unity only within narrow time windows. It also locates the deterministic process within specific frequency windows. Finally, it provides a further criterion to discriminate the filtered data set from its surrogate, which is not the case for other filtering techniques. These advantages propose our $\beta$-driven WT filter as the natural choice for brain signal.

$* * *$

The authors thank M. Peresson, V. Pizzella and F. Tecchio for help in the measurement session, and H. KANTZ for providing the software for the CD calculation.

\section{REFERENCES}

[1] Rapp P. E., Bashore T. R., Martinerie J. M., Albano A. M., Zimmermann I. D. and Mees A. I., Brain Topography, 2 (1989) 99.

[2] Basar E., Chaos in Brain Functions (Springer, Berlin-Heidelberg-New York) 1990.

[3] Pritchard W. S. and Duke D. W., Int. J. Neurosc., 67 (1992) 31.

[4] Pritchard W. S., Duke D. W., Coburn K. L., Moore N. C., Tucker K. A., Jann M. W. and Hostetler R. M., Electroencephalogr. Clin. Neurophysiol., 91 (1994) 118.

[5] Schiff S. J., Jerger K., Duong D. H., Chang T., Spano M. L. and Ditto W. L., Nature, 370 (1994) 615.

[6] Pijn J. P., Van Neerven J., Noest A. and Lopez da Silva F. H., Electroencephal. Clin. Neurophysiol., 79 (1991) 371.

[7] Ackermann P., Hartmann R., Gunzinger A., Guggenbühl W. and Borbely A. A., Electroencephal. Clin. Neurophysiol., 90 (1994) 384.

[8] Boccaletti S., Giaquinta A. and Arecchi F. T., to be published Phys. Rev. E, (May 1997).

[9] Arecchi F. T., Basti G., Boccaletti S. and Perrone A. L., Europhys. Lett., 26 (1994) 327.

[10] Grassberger P. and Procaccia I., Physica D, 9 (1983) 189.

[11] Storm Van Leeuwen W., Electroencephal. Clin. Neurophysiol. Suppl., 34 (1977) 1.

[12] Badi R., Broggi G., Derighetti B., Ravani M., Ciliberto S., Politi A. and Rubio M. A., Phys. Rev. Lett., 60 (1988) 979.

[13] Ruelle D., Proc. R. Soc. London, Ser. A, 427 (1990) 241.

[14] Kantz H. and Schreiber T., Chaos, 5 (1995) 143.

[15] Foglietti V., Del Gratta C., Pasquarelli A., Pizzella V., Torrioli G., Romani G. L., Gallagher W. J., Ketchen M. B., Kleinsasser A. W. and Sandstrom R. L., IEeE Trans. Magn., 27 (1991) 2959.

[16] Takens F., in Lect. Notes Math., Vol. 898 (Springer Verlag, New York) 1980, p. 365.

[17] Fraser A. M. and Swinney H. L., Phys. Rev. A, 33 (1986) 1134.

[18] Daubechies I., IEEE Trans. Inf. Theory, 36 (1990) 961.

[19] Daubechies I., Wavelets (SIAM, Philadelphia) 1992.

[20] Theiler J., Eubank S., Longtin A., Galdrikian B. and Farmer J. D., Physica D, 58 (1992) 77.

[21] Kennel M. B., Brown R. and Abarbanel H. D. I., Phys. Rev. A, 45 (1992) 3403. 